\title{
Bayesian Optimization for Radio Resource Management: Open Loop Power Control
}

\author{
Lorenzo Maggi, Alvaro Valcarce, Senior Member, IEEE, and Jakob Hoydis, Senior Member, IEEE
}

\begin{abstract}
We provide the reader with an accessible yet rigorous introduction to Bayesian optimisation with Gaussian processes (BOGP) for the purpose of solving a wide variety of radio resource management (RRM) problems. We believe that BOGP is a powerful tool that has been somewhat overlooked in RRM research, although it elegantly addresses pressing requirements for fast convergence, safe exploration, and interpretability. BOGP also provides a natural way to exploit prior knowledge during optimization. After explaining the nuts and bolts of BOGP, we delve into more advanced topics, such as the choice of the acquisition function and the optimization of dynamic performance functions. Finally, we put the theory into practice for the RRM problem of uplink open-loop power control (OLPC) in 5G cellular networks, for which BOGP is able to converge to almost optimal solutions in tens of iterations without significant performance drops during exploration.
\end{abstract}

Index Terms-Radio resource management, Bayesian optimization, Gaussian processes, Uplink power control, Machine Learning

\section{INTRODUCTION}

The current generations of cellular networks (4G \& 5G) specify a plethora of options and parameters to adapt them to different frequency bands and a wide range of deployment scenarios. However, how to choose these parameters optimally when deploying and operating a commercial network is not specified. This problem is generally referred to as radio resource management (RRM), whose complexity has grown from generation to generation. Such trend is likely to continue for $6 \mathrm{G}$ which is expected to serve an even larger diversity of spectrum, deployment options, and use cases [1]. In order to deal with this growing complexity, machine learning (ML) methods are seen as a key technology and, consequently, a large number of vision and survey papers have been published during the last few years on this topic, e.g., [2], [3]. Yet, several challenges to the use of ML for RRM in commercial networks are frequently pointed out [4], [5]: convergence speed, safe exploration and interpretability of solutions.

The first challenge relates to the time or number of trials it takes to find a good set of parameters. This is not an issue in simulation-based studies, but of utmost importance for realistic deployments. While reinforcement learning (RL) has shown a great potential to solve RRM problems in simulations by running algorithms for thousands of iterations (e.g., [6]), it often suffers from poor convergence speed which renders its use questionable for many real-world applications, where one is often limited to a few tens of iterations. The

The authors are with Nokia Bell-Labs France, Route de Villejust, 91620 Nozay, France (Email: \{lorenzo.maggi, alvaro.valcarce_rial, jakob.hoydis\}@nokia-bell-labs.com). second challenge is to avoid that an ML algorithm chooses RRM parameters that lead to unacceptably low performance levels, even if only chosen temporarily as an intermediate step towards better settings. Again, while this is not an issue in simulations, it is another key requirement of mobile network operators (MNOs) which will ultimately decide if a solution is adopted in practice or not. The last challenge relates to understanding why an ML algorithm has chosen a specific parameter setting. This is not necessarily an issue when the first two points are well addressed, but certainly of help for debugging purposes and to increase trust in ML methods in general.

Although these challenges are widely known and discussed, it is surprising that little research is done to explore the use of ML methods which are better suited to such practical constraints. In our experience, Bayesian optimisation with Gaussian processes (BOGP) ${ }^{1}$ is a very powerful tool, which is well adapted to a wide range of RRM parameter tuning tasks, and that addresses the above challenges to a large extent. As shown recently in [7] for the task of transmit power and antenna tilt optimization, BOGP can achieve convergence within two orders of magnitude fewer iterations than a modern RL-based solution. A remarkable additional advantage of BOGP is that it allows to naturally embed prior knowledge from experts, simulations, or real-world deployments in the optimization process. The basics of BOGP are already described in [8]. In addition, our paper addresses the difficulties that BOGP practitioners will encounter when applying it to RRM problems. This includes topics such as prior reuse for faster convergence, time evolving objective functions and the choice of a suitable acquisition function.

The goal of this paper is hence two-fold. First, we aim to provide an intuitive yet detailed user guide to Bayesian optimization (BO) with Gaussian processes (GPs). Second, we claim that BO is a useful but yet largely untapped algorithmic tool for RRM in general, and we demonstrate it via a specific use-case, namely that of open-loop power control (OLPC) in the uplink. In Section II] we aim to give the reader the ability to understand whether the RRM problem at hand can/should be solved via BO. Then, if the answer is positive, we invite the reader to embark on a more technically involved journey through the fundamentals of the theory of GPs and BO in Section III Section IV describes aspects of BOGP for RRM problems, while Section $\square$ focuses explicitly on OLPC. The paper is concluded in Section VI.

\footnotetext{
${ }^{1}$ Throughout the paper, we employ indifferently the acronyms BOGP and BO (Bayesian optimization).
} 


\section{IS BAYESIAN OPTIMIZATION SUITED FOR YOUR RADIO RESOURCE MANAGEMENT PROBLEM?}

It is convenient at this early stage to outline the general RRM framework that we will refer to throughout the paper, and that we will instantiate via uplink power control ${ }^{2}$

RRM problem: There exists a set of $k$ parameters, described by the vector $\boldsymbol{x} \in \mathbb{R}^{k}$, that a radio system hinges upon and that a controller wants to optimize. We call $\mathcal{X} \subset \mathbb{R}^{k}$ the set of available parameter configurations. We remark that $\mathcal{X}$ can be a discrete set. The performance of the system, when the parameters take on the value $\boldsymbol{x}$, is described by a single real value $f(\boldsymbol{x}) \in \mathbb{R}$. The function $f($.$) is unknown$ to the controller and is possibly noisy. We call $\widetilde{f}($.$) its$ realizations, and suppose $\mathbb{E}[\tilde{f}()]=.f($.$) . The controller$ is allowed to sequentially test different parameter values $\boldsymbol{x}(1), \boldsymbol{x}(2), \ldots$, and it is able to observe the corresponding performance realizations $\widetilde{f}(\boldsymbol{x}(1)), \widetilde{f}(\boldsymbol{x}(2)), \ldots$ The general goal is to ensure that performance is good at any point in time, while possibly converging to the optimal configuration $\boldsymbol{x}^{*}=\arg \max _{\boldsymbol{x}} f(\boldsymbol{x})$ and, most importantly, without incurring sudden drops in performance.

A more difficult variant of this problem arises when the function $f($.$) varies over time:$

Dynamic RRM problem: Assume that at any time $t$, there exists a hidden system "state" $\theta(t)$ that varies over time independently of the deployed RRM configuration. Then, the system performance is a function $f(\boldsymbol{x}, \theta(t))$ of the hidden state. When the controller deploys at time $t$ the configuration $\boldsymbol{x}$, it only observes $f(\boldsymbol{x}, \theta(t))$ but it is agnostic to $\theta(t)$.

We deliberately decide not to make the concept of "state" $\theta$ explicit at this stage. One can think of $\theta$ as the collection of random environmental variables that evolve exogenously (e.g., user geographical distribution, cell load, etc.), that affect the system performance, and that are hard to identify $a$ priori. We will come back to the dynamic RRM problem in Section III-C, where our approach does not prescribe to infer $\theta(t)$ itself, but rather to learn its speed of variation-and if any, its periodicity - and track the time-varying maximum of the function $f(., \theta(t))$.

\section{A. What must hold for BO to be a suitable choice}

In order for $\mathrm{BO}$ to be considered as a viable solution for the general RRM problem defined above, the latter must fulfill two main conditions. We argue that the first condition is the most binding one.

C1. Number of control parameters: The theory of BO is general enough to account for any (finite) number $k$ of parameters to be jointly optimized. In practice, however, $k$ must be "small", i.e., of the order of 20 as a rule of thumb [9]. As explained in Section IV, the motivation is that a nonconvex problem in $\mathbb{R}^{k}$ has to be solved for choosing the next RRM configuration to be deployed in the system.

\footnotetext{
${ }^{2}$ Although our focus is on RRM problems, most of the following discussion is also valid for the broader class of resource management problems.
}

C2. Smoothness of performance function: The performance function $f$ must not have discontinuities with respect to the control parameters $\boldsymbol{x}$. Indeed, BO strongly leverages the assumption that the function $f$ is sufficiently smooth to be able to infer it everywhere (i.e., for all possible values of $\boldsymbol{x}$ ) by just sampling it at a (possibly) small number of points.

Although the theory falls apart when the performance function $f$ presents discontinuities ( $\mathrm{C} 2)$, BO can still be used in practice, as long as the number of discontinuities is limited [10]. However, if the number of parameters is high (C1), we argue that $\mathrm{BO}$ is not a suitable tool for the RRM problem at hand.

\section{B. What should hold for BO to be a suitable choice}

We claim that there exists one additional-yet hard to quantify a priori-condition that should be met for BO to be successfully applicable to RRM problems.

C3. Update frequency: The control parameters $\boldsymbol{x}$ are to be modified at a relatively "low" frequency. It is hard to provide a rule of thumb for this, as it is dependent on the application. In general, it is a natural consequence of two factors:

i) At iteration $n$, once the parameter configuration $\boldsymbol{x}(n)$ is deployed in the system, the metric $\widetilde{f}(\boldsymbol{x}(n))$ is collected. Note that $\widetilde{f}(\boldsymbol{x})$ is meant as a sample average of the system performance computed while $\boldsymbol{x}$ is deployed in the system. The less noisy $\widetilde{f}($.$) is, the better the GP$ manages to infer the function $f($.$) , and the quicker \mathrm{BO}$ converges to the optimal configuration. Hence, updating $\boldsymbol{x}$ at a lower frequency allows one to observe and average out the performance metric across a longer time span, and eventually to improve the GP inference.

ii) At each iteration, deciding which point to try next is a task whose computational effort increases with the number of collected samples, and it generally requires to solve a non-convex problem. As a rule of thumb, for a reasonable range of applications with at most 100 different sampled configurations and 10 variables to optimize, one iteration may require a few seconds $3^{3}$

\section{The added value}

A prominent feature of $\mathrm{BO}$ consists in providing a principled approach to embed any prior information that the controller may possess on the shape of the performance function $f($.). Such prior can be expressed with two auxiliary functions.

C4. Mean prior: A prior function $m($.$) on the mean prior$ should reflect a "belief" on the unknown function $f$ at a point $\boldsymbol{x}$. Intuitively, one would want $m(\boldsymbol{x}) \approx f(\boldsymbol{x})$; by default, if no prior information is available, then one sets $m(\boldsymbol{x})=0$ for all possible values of $\boldsymbol{x}$.

C5. Covariance kernel: A prior function $C(.,$.$) on the$ covariance of $f$ should describe how much we expect the system performance to differ when the configurations $\boldsymbol{x}$ and $\boldsymbol{x}^{\prime}$ are deployed, respectively. In other words, one wishes that

\footnotetext{
${ }^{3}$ This depends of course on the available compute power.
} 
$C\left(\boldsymbol{x}, \boldsymbol{x}^{\prime}\right) \approx \operatorname{Cov}\left[f(\boldsymbol{x}), f\left(\boldsymbol{x}^{\prime}\right)\right]$. In practice, one should define $C\left(\boldsymbol{x}, \boldsymbol{x}^{\prime}\right)$ through kernel functions that only depend on the "distance" between $\boldsymbol{x}$ and $\boldsymbol{x}^{\prime}$ (see Section III).

Both the mean and the kernel are to be designed, e.g., by exploiting historical data or via expert domain knowledge. We remark that their specification is not necessary for a correct functioning of $\mathrm{BO}-$ in principle, they can be set to any default value-but it is still recommended if one wants to boost the convergence properties of the $\mathrm{BO}$ algorithm. Indeed, as we illustrate in Section $\mathrm{V}-\mathrm{C}$ via simulation, possessing a good prior mean function allows BO to avoid random exploration and sudden drops in system performance. On the other hand, as showed in Section III-D, the covariance kernel typically depends on hyperparameters that are optimized at run-time, as new observations are collected, which makes the prior choice for the covariance function $C$ less of a delicate task.

\section{The benefits of $B O$}

We now assume that conditions $\mathrm{C} 1-\mathrm{C} 2$ hold, and hopefully condition $\mathrm{C} 3$ is also verified. Then, we claim that $\mathrm{BO}$ can be successfully applied to solve the general RRM problem outlined at the beginning of this section. Moreover, the following properties can be appreciated which are particularly important for solving real-life problems:

P1. Quick convergence: BO converges to a near optimal configuration in few iteration steps.

P2. Safe exploration: BO avoids sudden drops in the experienced system performance since it searches across "safe" configuration parameter regions.

P3. Risk-vs.-return for untested configurations: $\mathrm{BO}$ is able to quantify the risk-vs.-return trade-off related to choosing any configuration parameter $\boldsymbol{x}$ (especially those which have never been deployed in the past).

We argue that the properties above are key in realistic RRM applications. They are also classic pain points for other optimization techniques which are more widely known in the RRM community, as illustrated next.

\section{E. Available alternatives to $B O$}

We now discuss alternatives to $\mathrm{BO}$ that are available in the literature and that could be employed to tackle our general RRM problem. We first observe that the controller is generally agnostic to the derivative of the performance function $f()-$. in fact, $f($.$) itself is supposed to be unknown - and can only$ observe its (noisy) samples at given configuration points. For this reason, the whole class of gradient descent optimization algorithms is not a viable option. Still, one could argue that the gradient could be approximated by finite differences. However, this clearly slows down convergence since $O(k)$ samples are used only for estimating the gradient, and moreover the observation noise can wildly corrupt the gradient estimate.

Turning to machine-learning (ML) based techniques that are widely known in the RRM community, multi-armed bandits (MAB) [11] could be in principle applied to our problem. E.g., one could discretize the parameter configuration space $\mathcal{X}$ and consider each available configuration as an independent arm.
This approach is agnostic to the derivative of $f($.$) , nonetheless$ it presents a major drawback in that MAB requires to sample each and every single arm at least once to accumulate sufficient statistics, i.e., it is not able to infer the quality of an RRM configuration that has never been tested. This is clearly unacceptable for real-world problems where convergence speed is key and properties P1-P2 are highly appreciated.

Another popular ML technique that is nowadays widely applied in the RRM space is Reinforcement Learning (RL) [12]. Yet, RL is particularly suited for time-varying reward (i.e., performance) functions where there exists a "state" that evolves according to exogenous and endogenous factors, i.e., it depends on the RRM control strategy of the controller as well. However, our primary focus is on static reward functions. Moreover, the time-varying RRM problem that we defined considers the evolution of the state $\theta(t)$ as independent of the RRM strategy, hence RL would be an overkill for our problem since there is no long-term strategic planning involved. Last but not least, it is widely known that RL generally suffers from serious convergence issues, for which it is inevitable to incur harsh performance drops during an - at least-initial exploration phase. Hence, this hinders us to achieve the qualitative convergence properties P1-P2. We remark that the RL community is of course aware of this issue and currently deploys important efforts to tackle it [13].

Let us turn our attention towards the realm of the socalled derivative-free optimization methods [14], which are completely agnostic to the function to be optimized. They can be classified into i) direct search methods, deciding the next point to sample only based on the value of the function at previously sampled points, without any explicit or implicit derivative approximation or function model building; ii) trustregion methods, defining a region around the current best solution, in which a (usually quadratic) model is built to approximate the unknown objective function and to decide the next point; iii) surrogate models, constructing a surrogate model of the unknown function at all points-hence not necessarily close to the current best solution.

Surrogate models are particularly suited when one can only afford to test a very small set of points before landing on a reasonably good solution. This typically occurs when the objective function is expensive to evaluate [15], e.g., for real-world applications like probe drilling for oil at given geographic coordinates, evaluating the effectiveness of a drug candidate, or testing the effectiveness of deep neural network hyperparameters. In our case, testing a new RRM configuration parameter is not expensive per se, but the controller still wants to greatly limit the exploration of new parameters and quickly converge to a reasonably good solution, leading to an equivalent concept of "expensive" objective function. BO is arguably the most prominent example of surrogate models, where the surrogate function is usually a GP. For this reason, we will next recap the theoretical foundations of GPs.

\section{Modeling THE PERformance FUnCTION AS A GAUSSIAN PROCESS (GP)}

In a nutshell, a GP is a collection of random variables, any finite number of which have a Gaussian distribution [16]. 
In this paper, we propose to model the system performance function $f($.$) as a GP, since GPs are a powerful inference$ engine allowing to predict the value of the function $f($.$) for$ RRM configurations that have never been deployed in the system. GPs are indeed the main sub-routine of BO [17], whose task is in turn to suggest to the controller the next configuration parameter to deploy in the network. This is achieved on the basis of the GP model that has been learned via the record of system performance observations resulting from the previously deployed RRM configurations.

Clearly, in practice the system performance function $f$ may follow a distribution which is not Gaussian. However, GPs have been shown to provide an excellent trade-off between modeling accuracy and inference complexity. For this reason, we will only focus on GPs as the main BO inference engine. Yet, it is worth mentioning that Student- $t$ processes are a valid alternative, as studied, e.g., in [18].

Before delving into the details of GPs, we deem it convenient to first review some basics on multivariate Gaussian distributions.

\section{A. Multivariate Gaussian distribution}

A random variable $Y$ with $d$ components is a Gaussian variable if its probability distribution $p($.$) can be written as$

$$
p(\mathbf{y})=\frac{1}{(2 \pi)^{d / 2}|\Sigma|} \exp \left(-\frac{1}{2}(\mathbf{y}-\mu)^{T} \Sigma^{-1}(\mathbf{y}-\mu)\right):=\mathcal{N}(\mu, \Sigma)
$$

where $\mathbf{y} \in \mathbb{R}^{d}, \mu \in \mathbb{R}^{d}$ is the mean vector of the random variable (i.e., $\left.\mu_{i}=\mathbb{E}\left[Y_{i}\right]\right), \Sigma$ is the covariance matrix (i.e., $\left.\Sigma_{i, j}=\mathbb{E}\left[\left(Y_{i}-\mu_{i}\right)\left(Y_{j}-\mu_{j}\right)\right]\right)$ and $|\Sigma|$ denotes the determinant of $\Sigma$. We remark that the diagonal terms of $\Sigma$ carry the variance of the each component, i.e., $\Sigma_{i, i}=\mathbb{E}\left[\left(Y_{i}-\mu_{i}\right)^{2}\right]$.

Conditioning: To understand GPs, it is crucial for the reader to be acquainted with the concept of conditioning for normal variables. Let us begin by splitting the components of $Y$ into two disjoint sets $A$ and $B$, and decompose the representation of their mean and covariance accordingly as:

$$
p\left(\left[\mathbf{y}_{A}, \mathbf{y}_{B}\right]\right)=\mathcal{N}\left(\left[\begin{array}{l}
\mu_{A} \\
\mu_{B}
\end{array}\right],\left[\begin{array}{c}
\Sigma_{A}, \Sigma_{A B} \\
\Sigma_{A B}^{T}, \Sigma_{B}
\end{array}\right]\right) .
$$

Now, let us suppose that we observe the realization $\mathbf{y}_{B}$ of the subset $B$ of the random variable $Y$, and we wish to infer the value of the remaining, hidden variables $A$. Intuitively, as the correlation between $A$ and $B$ increases, the uncertainty on $A$ after we observe $B$ decreases. This is formalized by the expression of the posterior probability $p\left(\mathbf{y}_{A} \mid \mathbf{y}_{B}\right)$ :

$$
p\left(\mathbf{y}_{A} \mid \mathbf{y}_{B}\right)=\mathcal{N}\left(\mu_{A}+\underline{\Sigma_{A B} \Sigma_{B}^{-1}\left(\mathbf{y}_{B}-\mu_{B}\right)}, \Sigma_{A} \underline{-\Sigma_{A B} \Sigma_{B}^{-1} \Sigma_{A B}^{T}}\right) .
$$

Without the observation $\mathbf{y}_{B}$, we could have simply computed the distribution of $\mathbf{y}_{A}$ alone as $p\left(\mathbf{y}_{A}\right)=\mathcal{N}\left(\mu_{A}, \Sigma_{A}\right)$. Thus, the underlined terms in (3) are correction factors in the mean and covariance of the variables $A$, brought by the knowledge of $\mathbf{y}_{B}$. Moreover, it is important to observe that in order to compute $p\left(\mathbf{y}_{A} \mid \mathbf{y}_{B}\right)$, the hardest bit is the inversion of the covariance matrix of the observations $\Sigma_{B}$, that can be performed in $O\left(\left(\text { size of } \mathbf{y}_{B}\right)^{3}\right)$.
Noisy observations: Suppose now that the observed samples $\mathbf{y}_{B}$ are noisy, i.e., they equal the true value of the realization plus an additive Gaussian noise with variance $\sigma^{2}$. Moreover, let us assume that the noise is independent across different components of the variables in $B$.

Since noise is itself a Gaussian variable then the resulting (noisy) random variable is still Gaussian. Moreover, by independence, the covariance $\Sigma$ stays unchanged, except for its diagonal terms that increase by a $\sigma^{2}$. Therefore, the case of noisy samples is accounted by maintaining the same formalism as above and just adding the noise term to the diagonal term of the covariance matrix, i.e., $\Sigma \leftarrow \Sigma+I \sigma^{2}$.

\section{B. GPs for RRM problems}

Formally speaking, a GP is an uncountable collection of random variables, such that any finite collection of those random variables has a multivariate Gaussian distribution. In our case, we will assume that the unknown performance function $f($.$) that the controller wants to maximize is modeled$ as a Gaussian process. Such an abstract concept leads in our practical case to the following situation. Assume that in the past, the controller has deployed in the system the parameter configurations $\boldsymbol{x}(1), \ldots, \boldsymbol{x}(n)$ and it has observed the corresponding performance metrics:

$$
\mathbf{o}(n)=[\widetilde{f}(\boldsymbol{x}(1)), \ldots, \widetilde{f}(\boldsymbol{x}(n))]^{T}
$$

where we recall that $\widetilde{f}($.$) is a noisy version of the actual$ (mean) performance metric $f($.$) . Then, by assumption, one$ can infer the performance function at any other value $\boldsymbol{x}$ via the GP posterior probability:

$$
\begin{aligned}
& p(f(\boldsymbol{x}) \mid \mathbf{o}(n))= \\
& \quad \mathcal{N}\left(\mu_{f}+\Sigma_{f \mathbf{o}} \Sigma_{\mathbf{o}}^{-1}\left(\mathbf{o}(n)-\mu_{\mathbf{o}}\right), \Sigma_{f}-\Sigma_{f \mathbf{o}} \Sigma_{\mathbf{o}}^{-1} \Sigma_{f \mathbf{o}}^{T}\right)
\end{aligned}
$$

where the equation above mimics the posterior probability for a Gaussian variable in (3).

One can already appreciate that GPs have the power to infer the value of the performance function for configurations that have never been deployed in the system. This feature really distinguishes BOGP from other approaches and it will reveal itself to be fundamental to avoid poor RRM decisions and converge "safely" to an optimal configuration.

Yet, in order to use effectively the posterior (5), we still have to define the mean vector $\mu$ and covariance matrix $\Sigma$ that we compute via the so-called prior mean and covariance functions that we describe next.

Prior mean function: To define the mean vectors $\mu_{f}, \mu_{\mathbf{o}}$ we resort to the concept of prior mean function $m():. \mathcal{X} \rightarrow \mathbb{R}$, that defines for each RRM configuration $\boldsymbol{x}$ the controller's prior belief $m(\boldsymbol{x})$ of the performance of the system.

Ideally, one would like $m(.) \approx f($.$) . By default, if no prior$ belief is available, one can safely set $m=0$ everywhere. In practice, such belief can be derived by crunching historical data, performing simulations, and/or interrogating human experts with domain knowledge. Another possibility is to define $m$ (.) via a parametric function [16], similarly to the covariance function as described next. This would however introduce a 


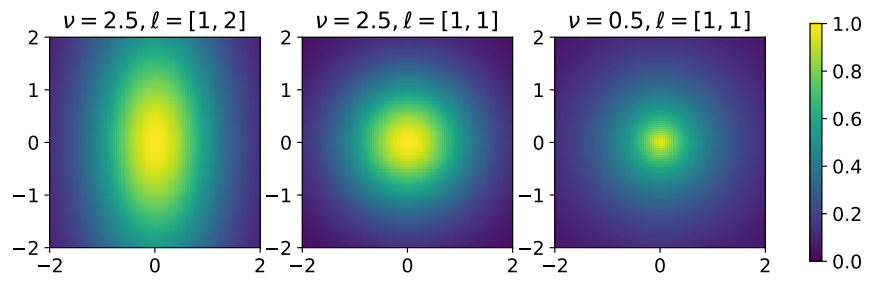

Fig. 1: Matérn kernel. Illustration of $K_{\theta}^{M}(\boldsymbol{x}, 0)$ for different values of the hyperparameters $\theta=[v, \ell]$, where $K^{M}$ is the Matérn kernel (7) and $\boldsymbol{x} \in \mathbb{R}^{2}$. The parameter $v$ controls the smoothness of the learned function, and via $\ell$ one can scale differently the control variables.

further complication that we do not deem necessary for our purposes. Hence, we will just assume $m($.$) as pre-determined$ and fixed. We provide further intuitions in Section $\mathrm{V}$, where we apply this framework to a practical RRM use case.

Covariance function: To define the covariance matrices $\Sigma_{f}, \Sigma_{\mathbf{0}}, \Sigma_{f_{\mathbf{0}}}$ in the GP posterior (5), we construct a function $C(.,):. \mathcal{X} \times \mathcal{X} \rightarrow \mathbb{R}$ that, informally speaking, describes how close we expect system performances obtained by deploying parameters $\boldsymbol{x}$ and $\boldsymbol{x}^{\prime}$ to be. Ideally, one would like $C\left(\boldsymbol{x}, \boldsymbol{x}^{\prime}\right) \approx \operatorname{Cov}\left(f(\boldsymbol{x}), f\left(\boldsymbol{x}^{\prime}\right)\right)$. In practice, $C(.,$.$) is usually$ defined via the so-called kernel functions $K_{\theta}$ that depend on the hyperparameters $\theta$. A basic example of kernel function is the Radial Basis Function (RBF), decreasing exponentially with respect to the distance between two candidate points, i.e.:

$$
K_{\theta}^{R B F}\left(\boldsymbol{x}, \boldsymbol{x}^{\prime}\right):=a \exp \left(-\frac{\left\|\boldsymbol{x}-\boldsymbol{x}^{\prime}\right\|}{2 b^{2}}\right) .
$$

The set of kernel hyperparameters is $\theta=[a, b]>0$, where $a, b$ regulate the amplitude and the smoothness of the objective function, respectively. The Matérn kernel is more sophisticated and writes:

$K_{\theta}^{M}\left(\boldsymbol{x}, \boldsymbol{x}^{\prime}\right):=\frac{1}{\Gamma(v) 2^{v-1}}\left(\sqrt{2 v} d_{\ell}\left(\boldsymbol{x}, \boldsymbol{x}^{\prime}\right)\right)^{v} \kappa_{v}\left(\sqrt{2 v} d_{\ell}\left(\boldsymbol{x}, \boldsymbol{x}^{\prime}\right)\right)$

where $d_{\ell}\left(\boldsymbol{x}, \boldsymbol{x}^{\prime}\right)=\sqrt{\sum_{i}\left(x_{i}-x_{i}^{\prime}\right)^{2} / \ell_{i}^{2}}, \theta=[v, \ell], \kappa_{v}$ is the modified Bessel function and $\Gamma$ is the Gamma function. Importantly, the parameter $v$ controls the smoothness of the learned function and one can scale differently the control variables via $\ell$, as shown in Fig. 1. For a more comprehensive list of kernel functions we refer to [16]. Then, by assuming that the noise affecting the observations is independent across different samples, the covariance function $C(.,$.$) is computed$ by adding to $K(.,$.$) the observation noise variance term \sigma^{2}$ only if $\boldsymbol{x}=\boldsymbol{x}^{\prime}$, i.e.,

$$
C_{\theta, \sigma}\left(\boldsymbol{x}, \boldsymbol{x}^{\prime}\right)=K_{\theta}\left(\boldsymbol{x}, \boldsymbol{x}^{\prime}\right)+\sigma^{2} \mathbb{I}\left(\boldsymbol{x}=\boldsymbol{x}^{\prime}\right) .
$$

In practical RRM applications, one should choose: i) the kernel function that is believed to suit best the shape of the system performance, and a reasonable initial value for both ii) the kernel hyperparameters $\theta$ and iii) the observation noise variance $\sigma^{2}$. Concerning i), we believe that the Matérn kernel (7) is sufficiently flexible for a large range of functions. Items ii) and iii) are greatly facilitated by the possibility of tuning the hyperparameters in real-time to make the covariance function "adhere" at best to the observations in a maximum-likelihood sense, as described in Section III-D

Computing the GP posterior (5): Once the prior mean $m$ and covariance function $C$ are defined, one can plug them in the expression of the GP posterior (5) as follows. We first define $\mu_{f}=[m(\boldsymbol{x})]$ as the prior mean for the point $\boldsymbol{x}$ at which performance is inferred. The prior mean vector at observed points $\mathbf{o}(n)$ is $\mu_{\mathbf{o}}=[m(\boldsymbol{x}(1)), \ldots, m(\boldsymbol{x}(1))]^{T}$. The covariance matrix $\Sigma_{\mathbf{0}}$ of the tested RRM configurations is such that $\left[\Sigma_{\mathbf{0}}\right]_{i, j}=C_{\theta, \sigma}(\boldsymbol{x}(i), \boldsymbol{x}(j))$ for $i, j=1, \ldots, n$. The (mono-dimensional) variance of the system performance $f(x)$ to be inferred at the yet-to-be-tested RRM configuration $\boldsymbol{x}$ writes $\Sigma_{f}=C_{\theta, \sigma}(\boldsymbol{x}, \boldsymbol{x})$. Finally, $\Sigma_{f \mathbf{o}}$ denotes the covariance column vector between $f(\boldsymbol{x})$ and the observations $\mathbf{o}(n)$, namely $\left[\Sigma_{f \mathbf{0}}\right]_{i}=C_{\theta, \sigma}(\boldsymbol{x}, \boldsymbol{x}(i))$.

\section{GPs for dynamic RRM problems}

The bulk of the literature on BOGP deals with the optimization of an unknown and noisy, yet static, function. This scenario fits well with our general RRM problem formulation in Section [I] However, in many practical situations, the system performance function to be optimized changes over time $t$, due to hidden environmental changes in the medium-long term, such as user spatial distribution and traffic intensity. To model this situation, in our dynamic RRM problem formulation in Section [I] we employed the concept of the hidden network "state" $\theta(t)$ that is assumed to evolve exogenously and to be independent of the controller's RRM strategy. Then, the performance function $f(.,$.$) itself is assumed to be dependent$ on both the RRM parameters $\boldsymbol{x}$ and the hidden state $\theta(t)$. In order to be consistent with the GP model, we discretize time and we call $t_{n}$ the time instant at the $n$-th time step.

Augmented observations: In order to model and track the time-varying function $f(\boldsymbol{x}, \theta(t))$ via the GP formalism, we follow an approach similar to the one proposed recently in [19]. We redefine our GP as a function of an augmented variable $\delta$ also including the time stamp of each observation:

$$
\delta(n):=\left[x(n), t_{n}\right], \quad \forall n \geq 1 .
$$

As we show next, this procedure lets the correlation between two samples depend also on the their time lag, hence allowing one to leverage seasonal patterns that may affect the hidden state $\theta$ to efficiently learn from past samples.

Covariance function: We now define a new GP whose covariance is decoupled as the product between the usual covariance function $C$, only depending on the "distance" between two RRM configuration, and a kernel function $K_{\theta^{1}}$ that only depends on the time lag between two samples. Then, the new covariance function writes:

$$
\begin{aligned}
C_{\theta^{\delta}, \sigma}\left(\boldsymbol{\delta}, \boldsymbol{\delta}^{\prime}\right)=C_{\theta, \sigma}\left(\boldsymbol{x}, \boldsymbol{x}^{\prime}\right) & \cdot K_{\theta^{1}}\left(t, t^{\prime}\right), \\
& \forall \boldsymbol{x}, \boldsymbol{x}^{\prime} \in \mathcal{X}, \forall t, t^{\prime} \geq 0
\end{aligned}
$$

where $\boldsymbol{\delta}=[\boldsymbol{x}, t], \boldsymbol{\delta}^{\prime}=\left[\boldsymbol{x}^{\prime}, t^{\prime}\right]$ and $\theta^{\delta}=\left[\theta, \theta^{1}\right]$. An interesting aspect of such a formulation for the RRM scenario is that the state $\theta$, although hidden and not always easily identifiable, often follows a periodic pattern which is usually strongly correlated with, e.g., the hour of the day or the day within 

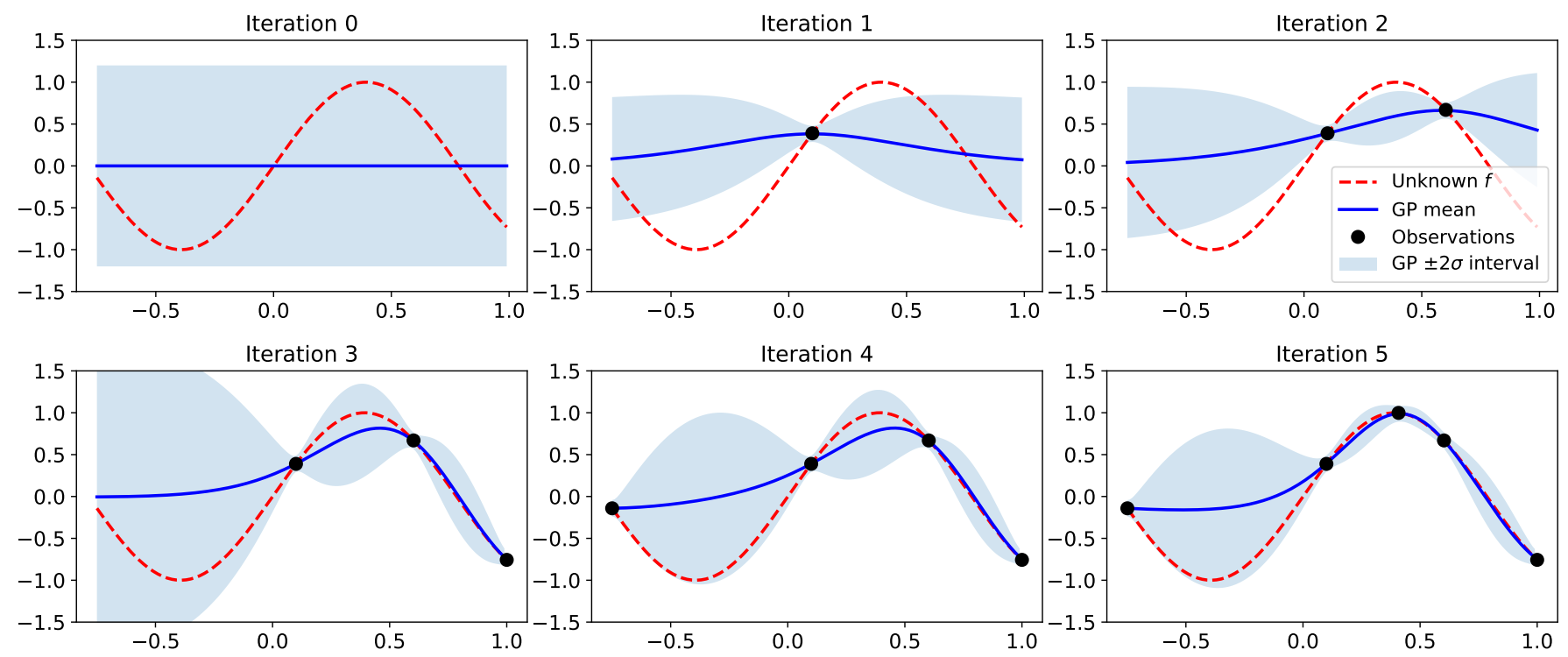

Fig. 2: Example of BOGP. The horizontal axis reports the value of the one-dimensional variable $\boldsymbol{x} \in \mathbb{R}$ to be optimized. The light blue shaded region and the blue line denote the confidence interval and the mean associated to the GP posterior computed as in (5), respectively. The red dashed line describes the true function $f($.$) . Black dots are the (noiseless) observations. A Matérn kernel K_{\theta}^{M}$ is used (see Eq. 7 and also Fig. 1. The GP starts at iteration 0 with mean prior $m=0$ everywhere.

the week. This holds especially if $\theta$ is influenced by the user activity and its geographic position. In such a case, a good choice for $K_{\theta^{1}}$ is the so-called Exp-Sine-Squared (aka periodic) kernel:

$$
K_{\theta^{1}}^{E S S}\left(t, t^{\prime}\right)=\exp \left(-\frac{2 \sin ^{2}\left(\pi\left|t-t^{\prime}\right| / D\right)}{\ell_{E S S}^{2}}\right)
$$

where $\theta^{1}=\left[D, \ell_{E S S}\right]$ is the vector of hyperparameters, $D$ describes the periodicity of the hidden state $\theta$ and the lengthscale $\ell_{E S S}$ regulates the covariance between samples whose time lag is not a multiple of the period $D$.

Mean prior function: Since the objective function $f$ is supposed to vary over time, it is reasonable to let the prior mean function $m$ depend on time as well. Then, $m(\boldsymbol{x}, t)$ is the system performance that one expects if the RRM configuration $\boldsymbol{x}$ is deployed at time instant $t$. Yet, as in the static case, such mean prior should be considered as a bonus: if such information is not available, one can settle for a constant prior mean $m(\boldsymbol{x}, t)=c$, for some arbitrary constant $c$.

Computing the GP posterior: Once the observations o( $n)$ are collected, we can infer the performance of any RRM configuration $\boldsymbol{x}$ at the next deployment time $t_{n+1}$ as:

$$
\begin{array}{r}
p\left(f\left(\boldsymbol{x}, \theta\left(t_{n+1}\right)\right) \mid \mathbf{o}(n)\right)=\mathcal{N}\left(\mu_{f}^{\delta}+\Sigma_{f \mathbf{o}}^{\delta} \Sigma_{\mathbf{o}}^{\delta^{-1}}\left(\mathbf{o}(n)-\mu_{\mathbf{o}}^{\delta}\right),\right. \\
\left.\Sigma_{f}^{\delta}-\Sigma_{f \mathbf{o}}^{\delta} \Sigma_{\mathbf{o}}^{\delta^{-1}} \Sigma_{f \mathbf{o}}^{\delta}\right)
\end{array}
$$

where $\mu_{f}^{\delta}=\left[m\left(\boldsymbol{x}, t_{n+1}\right)\right]$ is the prior mean at point $\boldsymbol{x}$ and at time $t_{n+1}$ and the vector of the observation means is $\mu_{\mathbf{o}}^{\delta}=\left[m\left(\boldsymbol{x}(1), t_{1}\right), \ldots, m\left(\boldsymbol{x}(n), t_{n}\right)\right]^{T}$. The covariance matrix $\Sigma_{\mathbf{0}}^{\delta}$ of the tested RRM configurations is such that $\left[\Sigma_{\mathbf{0}}^{\delta}\right]_{i, j}=C_{\theta^{\delta}, \sigma}(\boldsymbol{\delta}(i), \delta(j))$, whereas the (mono-dimensional) variance of the system performance $f(\boldsymbol{x})$ to be inferred at the yet-to-be-tested RRM configuration $x$ is $\Sigma_{f}^{\delta}=$
$\left[C_{\theta^{\delta}, \sigma}\left(\left[\boldsymbol{x}, t_{n+1}\right],\left[\boldsymbol{x}, t_{n+1}\right]\right)\right]$. Finally, $\Sigma_{f_{\mathbf{0}}}^{\delta}$ is the covariance column vector between $f\left(\boldsymbol{x}, \theta\left(t_{n+1}\right)\right)$ and the observations, namely $\left[\Sigma_{f \mathbf{o}}\right]_{i}=C_{\theta^{\delta}, \sigma}\left(\left[\boldsymbol{x}, t_{n+1}\right], \boldsymbol{\delta}(i)\right)$.

\section{Hyperparameter tuning}

We mentioned above that the covariance function $C$ is parametrized by the kernel hyperparameters $\theta\left(\theta^{\delta}\right.$, in the dynamic case; yet in this section we will stick to the static RRM notation for simplicity) and the observation noise variance $\sigma$. These are generally unknown to the controller, which has at best some prior belief $\theta_{0}$ and $\sigma_{0}$ on them. The GP framework offers a principled way to tune $\theta, \sigma$ in real-time, as new system performance observations $\mathbf{o}(n)$ are gathered. This is usually performed by finding those $\theta, \sigma$ that maximize the likelihood of the observed function values $\mathbf{o}(n)$, i.e.,

$$
\underset{\theta, \sigma}{\arg \max } p(\widetilde{f}(\boldsymbol{x}(1)), \ldots, \widetilde{f}(\boldsymbol{x}(n)))=\mathcal{N}\left(\mu_{\mathbf{0}}, \Sigma_{\mathbf{0}}\right)
$$

where $\mu_{\mathbf{0}}$ and $\Sigma_{\mathbf{0}}$ are the mean prior and covariance of the observations $\mathbf{o}(n)=[\widetilde{f}(\boldsymbol{x}(1)), \ldots, \widetilde{f}(\boldsymbol{x}(n))]^{T}$, respectively.

We remark that (13) is generally a non-convex problem, and one usually looks for a local maximum. Bayesian modelselection techniques for this goal are presented in [16], Chapter 5. More recent advances are proposed in [20], [21]. Otherwise, more standard gradient-descent optimization techniques can be used for (13), such as the Broyden-Fletcher-GoldfarbShanno (BFGS) algorithm [22], which is suited to general unconstrained and non-convex optimization problems.

\section{E. Dealing with a large number of observations}

The main computational bottleneck of the GP inference engine resides in the inversion of the covariance matrix of the observations, called $\Sigma_{\mathbf{0}}$ and $\Sigma_{\mathbf{0}}^{\delta}$ in the static and dynamic 
scenarios respectively, and used in (5), (12) for the computation of the GP posterior. At time step $n$, the matrix inversion requires $O\left(n^{3}\right)$ computations.

There exist methods in the literature to alleviate such issues, see, e.g., [16, Ch. 8]. These include two large classes of methods: i) approximating the covariance matrix as a matrix with reduced rank $n^{\prime}$, which allows one to use the Woodbury matrix identity, requiring the inversion of an $n^{\prime}$-by- $n^{\prime}$ matrix and ii) reducing the size of the set of observations by picking those maximizing a pre-defined significance criterion. Moreover, in the case of dynamic RRM, a sensible choice is to include in the observation set $\mathbf{o}(n)$ only the outcomes of the last $n^{\prime}<n$ tested RRM configurations, where $n^{\prime}$ should depend on the expected variation speed of the hidden state.

It is also worth mentioning that in the case of static RRM problems, the controller often cannot afford to explore among a large number of different configurations before sticking to a definitive choice. Thus, if the maximum envisioned number of tested configurations is less than $10^{3}$, the matrix inversion can be easily performed, e.g., via Cholesky decomposition [16].

\section{BO FOR RRM PROBLEMS}

The GP framework offers a powerful inference engine allowing one to predict, given the historical observations $\mathbf{0}(n)$, the current value of the system performance function for any possible RRM configuration. This is achieved via the posterior distribution formula (5) 4

At each step $n+1$, the role of BO is to leverage the GP inference based on observations $\mathbf{o}(n)$ and choose the next RRM configuration $\boldsymbol{x}(n+1)$ to be deployed in the system. We first observe that the BO's task is of strategic nature, and it is intimately connected with the Markov Decision Process (MDP) [23] control framework. In fact, if we define the control "state" as the information necessary to take the next decision $\boldsymbol{x}(n+1)$-in our case, the GP posterior (5) itself-then the controller alternates between i) taking a decision $\boldsymbol{x}(n+1)$ based on the current "state", ii) observing the associated random "reward" $\widetilde{f}(\boldsymbol{x}(n+1))$ and iii) experiencing a deterministic transition to a new "state" via the GP posterior formula. This procedure matches the MDP state-reward-transition formulation; however, the classic MDP algorithms (e.g., policy iteration, value iteration [23], or even more recent neural approximations [24]) are highly impractical in this scenario due to the astronomical size of the associated state space.

For such reason, BO takes a computationally simpler approach and optimizes at each step $n+1$ a so-called acquisition function $u(, \mid \mathbf{o}(n))$ that still depends on the previous observations via the GP posterior (5). The next selected RRM configuration $\boldsymbol{x}(n+1)$ is the one maximizing the acquisition function $u$ :

$$
\boldsymbol{x}(n+1)=\underset{\boldsymbol{x} \in \mathcal{X}}{\arg \max } u(\boldsymbol{x} \mid \mathbf{o}(n)) .
$$

The function $u$ should account for the exploitation-vs.exploration dilemma that naturally arises in such situations.

\footnotetext{
${ }^{4}$ In this section we will stick to the static RRM formulation. However, everything translates easily to the dynamic setting by just considering (12) as the GP posterior and by fixing the inference time to $t_{n+1}$.
}

In fact, the controller would want to sample a point with high expected performance, while minimizing the risk that the actual performance drops too low.

Although in the literature there exist several ways to define the acquisition function $u$ (e.g., see [17]), we will illustrate three relevant approaches for our purposes, called expected improvement and knowledge gradient and upper confidence bound. The first two ensure that the next configuration $\boldsymbol{x}(n+$ 1) in (14) is optimal for the MDP described above over a truncated time horizon, but under different assumptions on the observation noise.

Expected Improvement (EI): The EI is arguably the most widely known acquisition function. It was first introduced by [25] in a general Bayesian setting, and then applied to GPs in [26], [27]. To understand the theoretical motivations behind the EI, it is useful to perform the following thought experiment. Suppose that i) the observations are noiseless, i.e., $\widetilde{f}()=.f($.), ii) we are at time $n$, iii) at time $n+1$, we can test one more RRM configuration $\boldsymbol{x}(n+1)$, iv) at time $n+2$, we have to stick to one of the configurations already tested $\boldsymbol{x}(1), \ldots, \boldsymbol{x}(n+1)$, and $\mathrm{v})$ the experiment ends. Thus, knowing that at time $n+2$ we can always select the best point so far, the performance at time $n+$ 2 is $\max \left\{f(\boldsymbol{x}(n+1)), \max _{i=1, \ldots, n} f(\boldsymbol{x}(i))\right\}$. Therefore, the RRM choice $\boldsymbol{x}(n+1)$ that maximizes the expected performance at time $n+2$ is the one maximizing the expected improvement $u^{\mathrm{EI}}$ with respect to the best configuration so far, where:

$$
u^{\mathrm{EI}}(\boldsymbol{x} \mid \mathbf{o}(n))=\mathbb{E}\left[f(\boldsymbol{x})-\max _{i=1, \ldots, n} f(\boldsymbol{x}(i)) \mid \mathbf{o}(n)\right]^{+} .
$$

Note that $[.]^{+}=\max (., 0)$ is the positive part and the expected value is with respect to the GP posterior (5). A reason for its popularity is that $u^{\mathrm{EI}}$ has a closed form for GPs that writes:

$$
\begin{aligned}
u^{\mathrm{EI}}(\boldsymbol{x} \mid \mathbf{o}(n))= & \left(\mathbb{E}[f(\boldsymbol{x}) \mid \mathbf{o}(n)]-\max _{i=1, \ldots, n} f(\boldsymbol{x}(i))-\xi\right) \Phi(Z)+ \\
& +\operatorname{Std}[f(\boldsymbol{x}) \mid \mathbf{o}(n)] \varphi(Z), \quad \forall \boldsymbol{x} \in \mathcal{X}
\end{aligned}
$$

where $\mathbb{E}[f(\boldsymbol{x}) \mid \mathbf{o}(n)]$ and $\operatorname{Std}[f(\boldsymbol{x}) \mid \mathbf{o}(n)]$ are the mean and standard deviations of the GP posterior, respectively; $\Phi$ and $\varphi$ are the cumulative and probability density function of the standard normal distribution, respectively, and $Z$ is defined as:

$$
Z=\frac{\mathbb{E}[f(\boldsymbol{x}) \mid \mathbf{o}(n)]-\max _{i=1, \ldots, n} f(\boldsymbol{x}(i))-\xi}{\operatorname{Std}[f(\boldsymbol{x}) \mid \mathbf{o}(n)]} .
$$

Importantly, $\xi$ denotes the amount of exploration during optimization. A risk-sensitive RRM controller will choose $\xi$ small (e.g., $\xi=0.01$ or even $\xi=0$ ) to minimize the risk of a performance drop during optimization. In fact, the EI offers a natural exploitation-vs.-exploration interpretation, since one wants to sample a point with a good trade-off between high expected performance (cf. exploitation) and low uncertainty (cf. exploration).

In practice, to solve (14) for the EI case, one can use firstor second-order methods. E.g., one suitable technique is to calculate first derivatives and use the quasi-Newton method L-BFGS-B as in [28].

It is important to remark that in our general RRM scenario, the acquisition function $u^{\mathrm{EI}}$ in $(15)$ is not well defined. In fact, it depends on the true value of the system performance 
function $f($.$) , while the controller can only actually estimate$ a noisy version $\widetilde{f}($.$) of it. A variety of heuristic approaches$ exist to deal with noisy observations [9]. For instance, one can simply replace $f($.$) with its noisy observation \widetilde{f}($.$) , else with$ the GP posterior mean $\mathbb{E}[f() \mid. \mathbf{o}(n)]$.

If the observation noise is dominating-especially if the RRM configuration is updated at a fast time scale, which does not allow the controller to have sufficient samples to estimate $f(x)$ well enough - then the performance of the EI degrades. In this case, one can adopt a different acquisition function that we describe next.

Knowledge Gradient (KG): The KG acquisition was first derived in [29] as a variant of EI that is particularly suited to the case of noisy observations of the performance function. $\mathrm{KG}$ can be defined in a constructive manner by recycling the thought experiment for EI above while modifying the assumptions i) and iv). We here suppose that i) observations are noisy and iv) at time $n+2$, one can choose any RRM configuration (even one that has not been tested in the past). Our goal is to choose $\boldsymbol{x}(n+1)$ at time $n+1$. If $\boldsymbol{x}(n+1)$ is selected with observation $\widetilde{f}(\boldsymbol{x}(n+1))$, then at step $n+2$, one would want to achieve the highest expected performance

$$
\mu^{*}(n+2)=\max _{\boldsymbol{x}^{\prime} \in \mathcal{X}} \mathbb{E}\left[f\left(\boldsymbol{x}(n+2)=\boldsymbol{x}^{\prime}\right) \mid \mathbf{o}(n), \widetilde{f}(\boldsymbol{x}(n+1))\right] .
$$

Equivalently, the controller would seek to maximize the improvement of system performance $\mu^{*}(n+2)-\mu^{*}(n+1)$, where we analogously define $\mu^{*}(n+1)=\max _{x \in \mathcal{X}} \mathbb{E}[f(\boldsymbol{x}) \mid \mathbf{o}(n)]$. Yet, at time $n$, the value of $\widetilde{f}(\boldsymbol{x}(n+1))$ is unknown and $\mu^{*}(n+2)-\mu^{*}(n+1)$ a random quantity. Hence, we take its expectation to finally compute the $\mathrm{KG}$ acquisition function:

$$
u^{\mathrm{KG}}(\boldsymbol{x} \mid \mathbf{o}(n))=\mathbb{E}\left[\mu^{*}(n+2)-\mu^{*}(n+1) \mid \boldsymbol{x}(n+1)=\boldsymbol{x}\right] .
$$

Although KG performs better than EI in the noisy observation scenario, it still suffers from computational issues since there exists no closed-form expression for (19) and one has to resort to evaluate it via simulations. We remark that an efficient and scalable approach, based on multi-start stochastic gradient ascent, has been proposed in [30].

Upper Confidence Bound (UCB): Both EI and KG aim at improving the value of the objective function with respect to the best observation so far. Yet, the objective function itself may vary over time with the state $\theta$, as in Section III-C. Thus, at each step $n$ EI and $\mathrm{KG}$ should in principle compute the best observation among all those where the network found itself in the same state $\theta=\theta\left(t_{n}\right)$, and define the improvement accordingly. This clearly poses a challenge, since the state $\theta$ is generally hidden or hard to infer. For this reason, in the dynamic scenario, it is more convenient to employ the UCB acquisition function [31], that only depends on the GP posterior and not on previous observations. UCB naturally trades off exploration and exploitation, i.e., choosing points with high GP posterior variance and mean, respectively, via the coefficient $\beta_{n} \geq 0$ :

$$
u(\boldsymbol{x} \mid \mathbf{o}(n))=\mathbb{E}[f(\boldsymbol{x}) \mid \mathbf{o}(n)]+\beta_{n} \operatorname{Std}[f(\boldsymbol{x}) \mid \mathbf{o}(n)] .
$$

The closed form expression of $u^{\mathrm{UCB}}$ directly stems from the GP posterior definitions in [5, (12). We refer to [31] for the tuning of coefficients $\beta_{n}$ 's.

Safe exploration and termination: It should be now apparent that one of the great advantages of the GP resides in the possibility of evaluating the risk-vs.-benefit trade-off associated with any possible RRM configuration. In turn, via the optimization of the acquisition function, BO allows the controller to fully exploit the information provided by the GP inference engine to improve the system performance and, remarkably, to avoid to deploy configurations that are inferred to perform poorly with high probability.

In the static RRM scenario, the BO framework also allows the controller to naturally figure out when it is reasonable to terminate the exploration and eventually stick to a fixed RRM configuration. For instance, the exploration may stop when the achievable expected improvement of the system performance is lower than a threshold $\epsilon$, i.e.,

$$
\max _{\boldsymbol{x} \in \mathcal{X}} u(\boldsymbol{x} \mid \mathbf{o}(n))<\epsilon
$$

where $u:=u^{\mathrm{EI}}$ or $u:=u^{\mathrm{KG}}$. Otherwise, the controller could simply set a maximum number $\bar{n}$ of exploration steps. Afterwards, the controller would stick to the RRM configuration $\boldsymbol{x}^{*}=\arg \max _{\boldsymbol{x}(i), i=1, \ldots, n} \widetilde{f}(\boldsymbol{x}(i))$ that allowed the system to achieve the highest performance so far.

We recap the main steps of BOGP for static and dynamic RRM problems in Algorithms 1 and 2, respectively. We show in Fig. 2 a visual insight of the BOGP behavior for one optimization variable. In the next section, we illustrate the benefits of adopting the BOGP framework for the optimization of network-wide system parameters in a specific uplink power control RRM use-case.

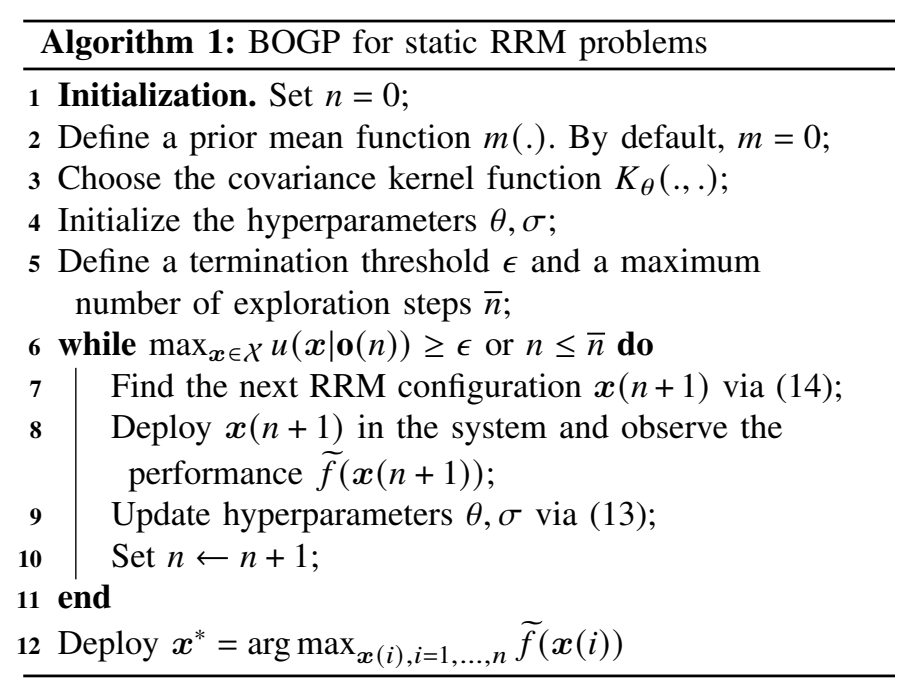

\section{OPEN LOOP POWER CONTROL}

In this section, we consider a practical instance of the general RRM problem defined in Section III, namely uplink power control (ULPC), which consists in managing the transmit power of UEs in cellular networks. In Long Term Evolution (LTE) and 5G New Radio (5GNR), the transmissions of 

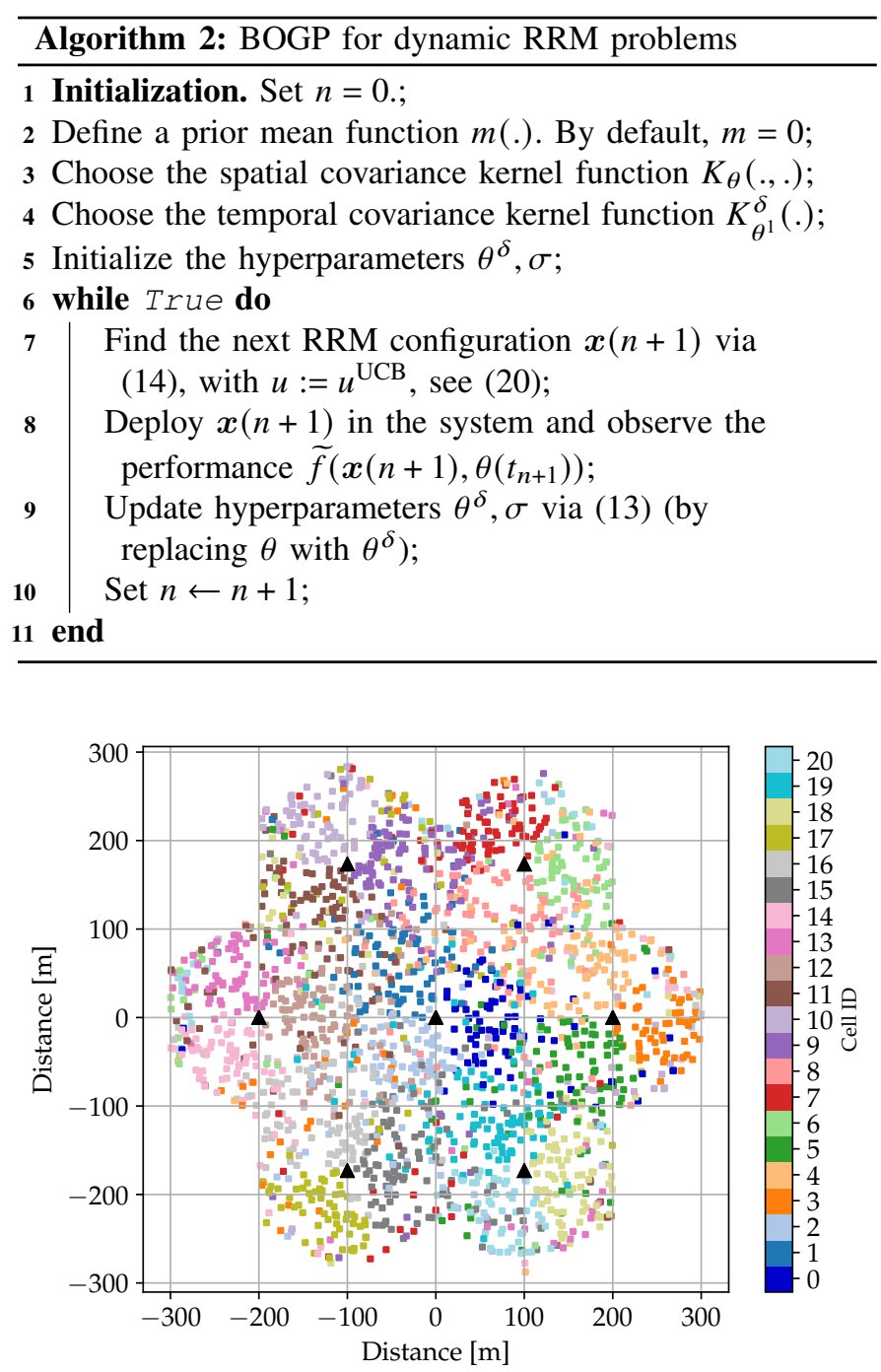

Fig. 3: Network layout. Triangles denote base station (BS) sites, squares describe user equipment (UE) locations, and their color characterizes their serving cell.

different UEs within the same cell must reach the BS with approximately the same power. This is important to manage the average cell spectral efficiency, as well as user throughput fairness. For cell-edge UEs, the same transmit power spectral density would cause more interference to neighbor cells when compared to cell-center UEs. For this reason, 3GPP adopts the so-called fractional power control approach, where the uplink transmit power compensates only for a fraction of the total pathloss.

As per 3GPP TS 38.213, UEs calculate their physical uplink shared channel (PUSCH) transmission power $P_{P U S C H}$ in $\mathrm{dBm}$ as follows:

$$
P_{P U S C H}=\min \left\{P_{C M A X}, P_{0}+10 \log \left(M_{R B}\right)+\alpha P L+C L\right\}
$$

where $P_{C M A X}$ is the UE configured maximum output power, $M_{R B}$ is the number of physical resource blocks (PRBs) allocated to the UE, $\alpha$ is the fractional power control compensation parameter, $P L$ is the downlink pathloss estimate, $C L$ is the

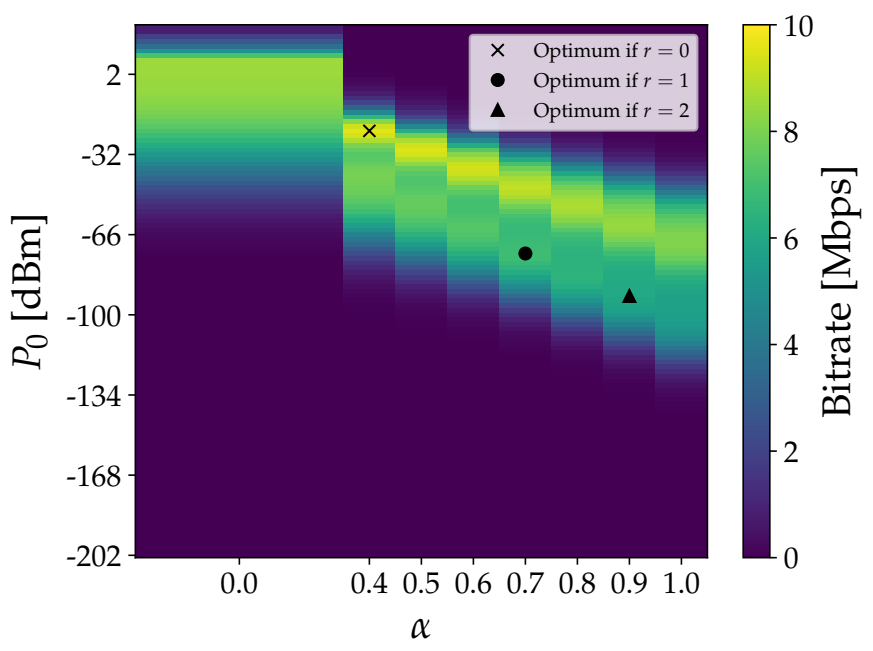

Fig. 4: System performance function. Utility function $u\left(\alpha, P_{0}\right)$ with fairness $r=0$ on the $\left(\alpha, P_{0}\right)$ solution space when simulated with $K=4$ UEs. The optimum $x^{*}=\left(\alpha^{*}, P_{0}^{*}\right)$ for other values of $r$ are also shown.

closed-loop power control adjustment and $P_{0}$ is the power per PRB that would be received under full pathloss compensation.

UEs transmitting with power higher than needed will experience battery drain and cause unnecessary inter-cell interference to the uplink of nearby cells. On the other hand, UEs transmitting with low powers will have a poor Quality of Experience or even worse, connectivity losses. A proper management of the transmit powers of the uplink data channels is therefore essential for the network to operate well.

Open-loop power control (OLPC) refers to the selection of slow-changing parameters that UEs can use to autonomously select their own transmit power. As per (22), the parameters that control this behavior on each network cell are $P_{0}$ and $\alpha$. The selection of values (see Table If) for these parameters defines the uplink inter-cell interference operation point and hence the average uplink radio performance. Closedloop power control (CLPC) then applies fast transmit power corrections around this point.

TABLE I: Allowed values for the 3GPP OLPC parameters

\begin{tabular}{|r|l|}
\hline$P_{0}$ & $-202,-200, \ldots,+22,+24$ \\
\hline$\alpha$ & $0,0.4,0.5,0.6,0.7,0.8,0.9,1$ \\
\hline
\end{tabular}

As of 2021, most MNOs choose a set of default values for the OLPC parameters and then update individual cells ondemand, following technical intuitions. However, the inter-cell interference behavior of a cellular network is not intuitive at all, which makes this procedure costly and sub-optimal.

Exhaustive search approaches are simply not scalable due to the combinatorial nature of the problem. In fact, there are eight possible standard values of $\alpha$ and 114 possible values for $P_{0}$, hence, a total of $N=8 \cdot 114=912$ possible OLPC configurations for any given cell in a network. In a network with $C$ cells, there are $N^{C}$ possible OLPC network configurations. As an example, this rises to nearly 760 million combinations even for a small network with three cells. Importantly, some 
OLPC configurations yield very low uplink performance (see [32], [33]). These are unknown a priori and are to be avoided. Testing these configurations for the sake of exploration is usually unacceptable to MNOs, which is why methods are needed to optimize the OLPC configuration in few steps while exploring safely.

As in most RRM problems, the relationships between the OLPC parameters and the uplink key performance indicators (KPIs) are complex and hard to model. For this reason, we propose to treat this dependence as a black-box and optimize it via a Bayesian approach, with a Gaussian process modeling the performance function based on the preferred system KPI. A main advantage of this approach is that domain knowledge and/or system simulation results can be exploited to design an initial prior of the performance function, allowing one to significantly increasing the safety and convergence speed of the exploration procedure. Next we provide details about this approach.

\section{A. Optimization target}

Most networks monitor performance and provide cell-level KPIs periodically (e.g., every 15 minutes). Examples of UEspecific uplink KPIs include signal-to-noise-plus-interference ratio (SINR), block error rate (BLER), bitrate, etc. Our iterative Bayesian optimization (BO) approach collects these samples from all network cells and processes them in a centralised self organizing networks (C-SON) server. Then, based on the data collected, it proposes a new OLPC configuration for each and all network cells.

Let $\boldsymbol{x}=\left(\alpha, P_{0}\right)$ be an OLPC configuration containing the values of the $\alpha$ and $P_{0}$ parameters for each network cell. Then, let $\gamma_{c, u}(\boldsymbol{x})$ denote the PUSCH SINR of UE $u$ in cell $c$ when the network uses configuration $x$. Let $B_{R B}$ denote the bandwidth of one PRB and let $N_{c, u}$ be the number of uplink PRBs scheduled for UE $u$ in cell $c$. The bitrate of UE $u$ in cell $c$ can either be obtained from network traces or approximated by the Shannon formula:

$$
R_{c, u}(\boldsymbol{x})=N_{c, u} \cdot B_{R B} \cdot \log _{2}\left(1+\gamma_{c, u}(\boldsymbol{x})\right) .
$$

To capture the trade-off between cell-edge and cell-center UE performance, we apply an alpha-fairness function (see [34]) to the collected KPI samples. Alpha-fairness can be applied to any uplink KPI (e.g., SINR, bitrate, etc). For example, when applied on bitrate samples, the alpha-fairness metric is

$$
\Omega^{(r)}\left(R_{c, u}(x)\right)= \begin{cases}10 \cdot \log _{10}\left(R_{c, u}(x)\right), & r=1 \\ \frac{R_{c, u}(\boldsymbol{x})^{1-r}}{1-r}, & r \neq 1\end{cases}
$$

where $r \geq 0$ is the fairness parameter.

The objective of OLPC performance optimization is the maximization of a network-wide utility, which we define as the expected alpha-fairness metric perceived by a UE with respect to a certain UE distribution $\rho$ :

$$
f(\boldsymbol{x})=\mathbb{E}_{\rho}\left[\Omega^{(r)}\left(R_{c, u}(\boldsymbol{x})\right)\right]
$$

which in practice is approximated via a sample average. The optimization problem can then be formulated as:

$$
\boldsymbol{x}^{*}=\underset{x}{\arg \max } f(\boldsymbol{x}) .
$$

It is worth noting that different levels of the fairness parameter $r$ yield optimization problems that are equivalent to optimizing the different Pythagorean means:

$$
\boldsymbol{x}^{*}=\underset{x}{\arg \max } f(\boldsymbol{x})= \begin{cases}\arg \max _{x} A M\left(R_{c, u}(\boldsymbol{x})\right), & r=0 \\ \arg \max _{x} G M\left(R_{c, u}(\boldsymbol{x})\right), & r=1 \\ \arg \max _{x} H M\left(R_{c, u}(\boldsymbol{x})\right), & r=2\end{cases}
$$

where $A M, G M$, and $H M$ are the arithmetic, geometric, and harmonic means, respectively. Moreover, when $r \rightarrow \infty$ the configuration $\boldsymbol{x}^{*}$ tends to the max-min fair solution, for which any increase of the selected KPI for a user causes a KPI drop for a worse-off user. The $A M$ sensitivity to extreme values is well known, which is why a low level of fairness $(r=$ 0 ) maximizes uplink performance for the best-off UEs at the expense of UEs with lower bitrates. This sensitivity decreases with the fairness level $r$. Consequently, UEs with low bitrates are the ones that can profit the most from an optimization process with higher fairness.

We remark that, in practice, the KPIs measured by commercial networks are not stationary processes. Traffic demand and network usage often show seasonal patterns, at the time scale of day/week/year. Therefore, the samples collected by iterative techniques may age quickly and prevent convergence. This problem can be circumvented via dynamic RRM solutions that implicitly learn the seasonality of the traffic evolution. A possible simple solution is to capture the KPIs dependence on a heuristically defined internal network state (e.g., hour of day, traffic density, etc.) and train separate models for each such state.

\section{B. System architecture \& algorithm}

We propose to deploy a centralised self-organizing network (C-SON) server that collects cell-level uplink KPIs from the cells to be optimized and proposes a new OLPC configuration in return. The time duration during which KPIs are collected is called a sampling period and for scalability reasons, all cells are provisioned with the same OLPC configuration. We also experimented with grouping the cells into clusters and assigning different OLPC configurations to different clusters. However, this increased the size of the problem and convergence time by several orders of magnitude, in exchange for only marginal performance gains.

Our iterative BO algorithm would run in the C-SON server. At each sampling period $n$, it summarizes all the KPI samples collected during that period into the sample average $\widetilde{f}(\boldsymbol{x}(n))$ 25. This way, a database of configuration to performance mappings $\mathcal{D}=\{\boldsymbol{x}(n), \widetilde{f}(\boldsymbol{x}(n))\}_{\forall n}$ is progressively built and used to train a GP model of the system performance function. The configuration $\boldsymbol{x}(n+1)$ to try at the beginning of a new period $n+1$ is chosen such that an acquisition function is maximized as described in great detail in Section IV 


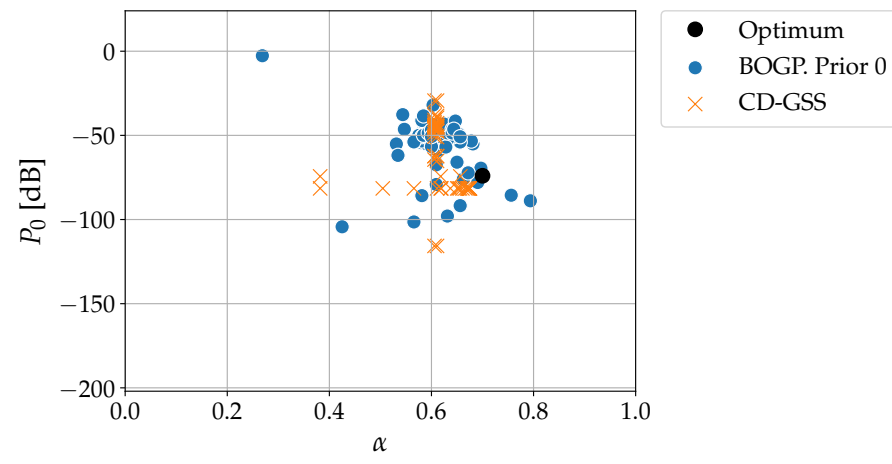

(a) OLPC configurations evaluated by the optimization algorithm.

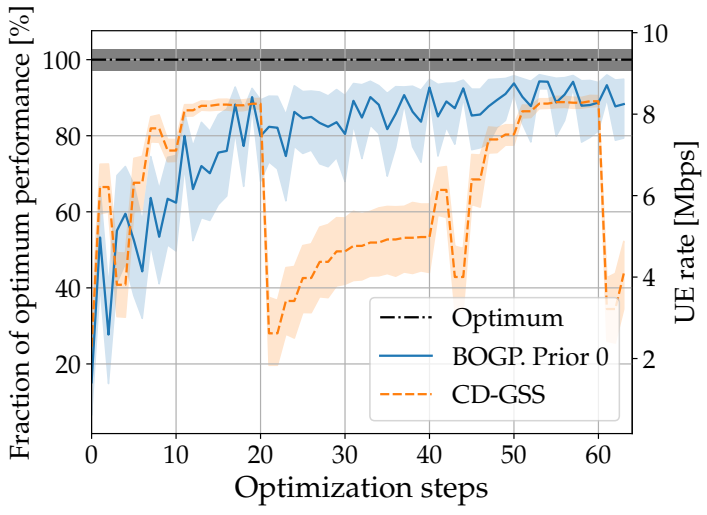

(b) Convergence time comparison. The solid line depicts the mean performance and the shadowed region the $95 \%$ confidence interval.

Fig. 5: BOGP convergence. Evaluation with $K=4$ active UEs per snapshot and a fairness level of $r=1$ on the network architecture of Fig. 3 The $\left(\alpha, P_{0}\right)$ solutions explored by BOGP with Prior 0 (see fig. 6b are shown. The performance of a coordinate descent $(\mathrm{CD})$ algorithm with golden-section search (GSS) on each coordinate is also reported for reference.

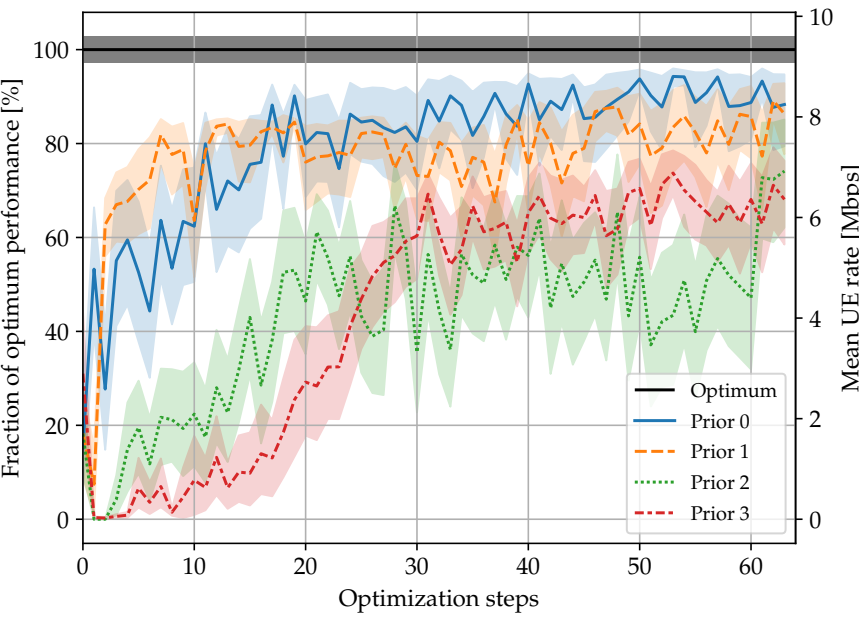

(a) BOGP convergence on a scenario with $N_{c}=21$ cells and 2100 UEs.
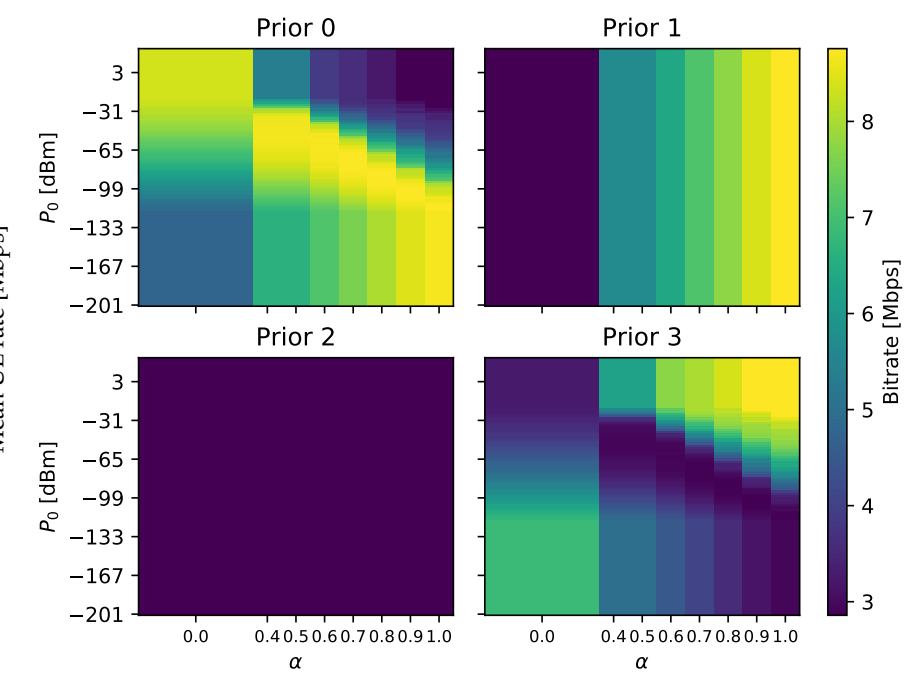

(b) Prior mean functions $m($.$) .$

Fig. 6: Impact of prior: Worst-case analysis. Examples of different priors obtained with a fairness level of $r=0$. Prior 0 has been built with 912 samples from a network with $N_{c}=3$ cells and 90 UE locations, whereas Prior 1 was built with only 10 samples. Prior 2 has zero mean. Prior 3 is the mirror of Prior 0. All priors share the same covariance matrix, which is a Matérn kernel function $K_{\theta}^{M}$ as defined in (7).

\section{Simulation setup \& Results}

We tested our algorithm in a simulated cellular network with $N_{\text {sites }}=7$ sites (see Fig. 3), $N_{\text {sectors }}=3$ cells per site, and $N_{b}=32$ beams per cell. There are $N_{c}=N_{\text {sites }}$. $N_{\text {sectors }}=21$ cells in total. The carrier frequency was $3.5 \mathrm{GHz}$ and the system bandwidth contained 100 physical resource blocks (PRBs) of bandwidth $180 \mathrm{kHz}$ each. The maximum transmit power per UE was $P_{C M A X}=23 \mathrm{dBm}$ and we used an urban micro (UMi) 3D path loss model based on Table 7.4.1-1 of 3GPP TR 38.901. In our network, $80 \%$ of UEs were indoor and $20 \%$ of UEs were outdoor and the line of sight (LoS) probability was computed as described in section 7.4.2 of 3GPP TR 38.901. We simulated a total of 2100 UEs, with an average of 100 UEs per cell, although not all UEs were connected to their serving cells all the time. A wrap-around radio distance model has been used to reduce interference bias for UEs at the edge of the network. At each snapshot, a set of $K \in[2, \ldots, 16]$ UEs per cell was sampled uniformly at random from the set of all UEs served by that cell. These parameter values have been chosen to simulate a network that is dense enough (both in terms of the number of cells and UEs) to be interference limited in the uplink. It is in such networks where the difference between poor and good power control can be observed more clearly. The power and spectral values resemble those from commercial systems.

At each sampling period, the base stations (BSs) were provisioned with a new OLPC configuration chosen by the BOGP algorithm. Then, $S=16$ snapshots were simulated 
with that configuration. $S$ reflects the number of performance management (PM) counters a C-SON server may be able to collect during a single day. This yields a total of $S \cdot N_{c} \cdot K$ uplink bitrate samples per sampling period. During each snapshot, the cells try to schedule PRBs equally to each of their served UE, while respecting their power limits (i.e., cell-edge UEs may not have enough uplink power to use as many PRBs as cellcenter UEs).

First, we used exhaustive search to explore the target function defined in (25) in the full $\left(\alpha, P_{0}\right)$ solution space. This produced the system performance function $f(x)$ illustrated in Fig. 4 which shows, among other traits, that $f$ is not unimodal. The locations of the optimum OLPC configurations for various fairness levels $r$ are also illustrated. Despite this being an idealized simulated scenario, i) the multi-modality of $f(x)$ and ii) the fact that only a noisy version $\widetilde{f}$ of it is observed already pose a challenge for traditional direct-search derivative-free algorithms (e.g., binary search, golden section search search).

Fig. 5 compares the performance of BOGP for fairness $r=1$ against that of the coordinate-descent Golden Section Search (CD-GSS) optimization algorithm [14], a well known directsearch derivative-free method that we use as a baseline. CDGSS optimizes over the $\alpha$ and $P_{0}$ coordinates in successive fashion, using GSS on each dimension. Within one coordinate pass and in the absence of observation noise, CD-GSS is capable to attain a local maximum with respect to the specific coordinate. However, after a coordinate change the performance drops suddenly, since a line search is performed from scratch with respect to the new coordinate. Indeed, we argue that direct-search methods are especially useful for offline derivative-free optimization, since they do not fulfill the properties P2-P3 outlined in Section II-D

In contrast, once BOGP has attained a near-optimal performance, it automatically reduces considerably the search region and avoids perilous and (almost) pointless further exploration. Moreover, BOGP achieves, on average, $90 \%$ of the optimum performance in barely 20 evaluations (see Fig. 5b). To achieve this, we used a covariance Matérn kernel function $K_{\theta}^{M}$ defined as in (7). We simply initialized the hyperparameters $\ell$ so as to homogenize the scale of the two variables of $x=\left[\alpha, P_{0}\right]$, i.e., $\ell_{\alpha} / \ell_{P_{0}}=226$. Fine tuning of the covariance kernel hyperparameter $\ell$ could possibly bring convergence closer to the optimum performance. Hyperparameter tuning can be done in simulation and prior to the actual optimization on a commercial network. However, fine-tuning $\ell$ on the live network would be hard to justify to an operator for a mere gain increase of $90 \%$ to $100 \%$. In this experiment, we employed the expected improvement (EI) acquisition function in its most risk-sensitive variant, i.e., with $\xi=0$.

Fig. $5 \mathrm{~b}$ shows the BOGP performance when the GP is initialized with a prior obtained from a different and smaller network with only $N_{c}=3$ cells and 90 UE locations for each $\left(\alpha, P_{0}\right)$ pair. Despite the major architectural differences and traffic requirements between the two networks, Fig. $5 \mathrm{~b}$ shows that prior reuse helps to improve performance in the initial steps and to quickly steer the optimization process towards a good $\left(\alpha, P_{0}\right)$ region. The solution regions explored by the BOGP are illustrated in fig. 5 a.

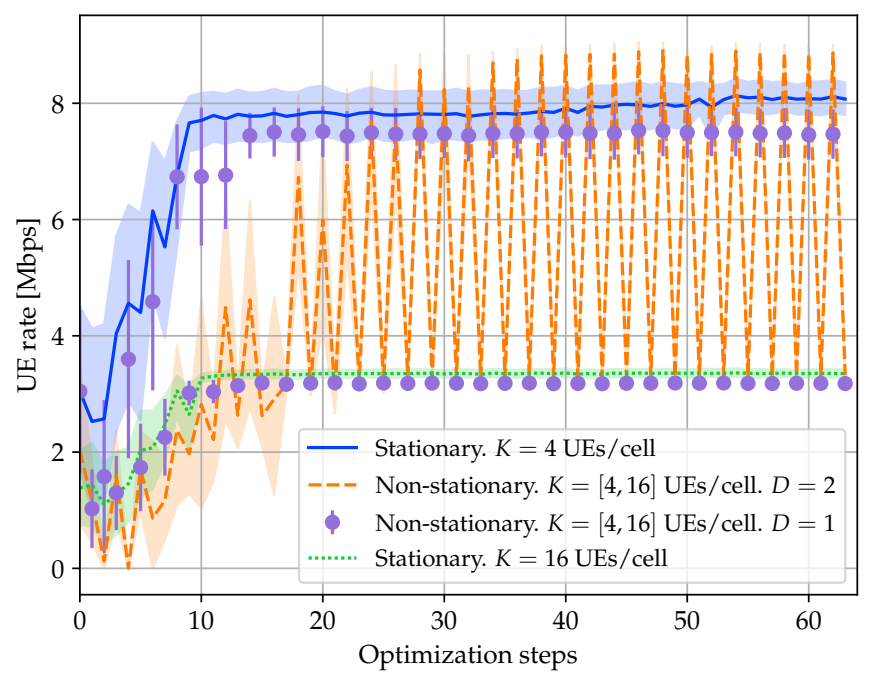

Fig. 7: Convergence in a load-varying scenario. In the stationary case, the number $K$ of active UEs per cell and TTI remains constant on all optimization steps. In the non-stationary case, it changes at every step (e.g., peak vs off-peak hours). The case $D=1$ coincides with the classic, non-dynamic BOGP algorithm.

No MNO would ever deploy an algorithm that drives uplink performance to 0 , even if high gains can be obtained later on. We therefore see the reuse of priors as one of the main advantages of the BOGP algorithm. In a real network setup, one could imagine that clusters of cells would share their priors to speed up the optimization of other clusters.

Finally, we illustrate in Fig. 6 the impact of using different, and possibly off-the-mark, priors when starting the optimization process. A fairness parameter $r=0$ is used here. As expected, priors with a mean close to that of the target function help to increase the performance very early in the optimization process (see Prior 0 in Fig. 6, obtained in the same 3 cell setup as above). As mentioned above, such priors can be obtained by collecting data from previous network deployments. Remarkably, even simplified priorssuch as Prior 1 which was obtained by interpolating Prior 0 on few samples-help to accelerate convergence, suggesting that the prior does not need to match accurately the actual performance function $f$. Rather, the prior should hint at the best regions that $\mathrm{BO}$ has to concentrate on.

As a worst-case analysis, priors that differ substantially from the target function (e.g., Prior 3 in Fig. 6, being the opposite of Prior 0) slow down convergence. In this case, $\mathrm{BO}$ is taught to focus on a low performance $\left(\alpha, P_{0}\right)$ region, which $\mathrm{BO}$ can escape only by random exploration, i.e., by setting the parameter $\xi>0$ and sufficiently high. Eventually, the algorithm is capable of learning the right trend and it overcomes the initial bad estimates, although the slow convergence may be unacceptable for most MNOs. Yet, we argue that this would constitute an extreme and unrealistic case. In fact, if the controller suspects that the available prior information is unreliable, then it should simply settle for a constant prior mean $m$ (see Fig. 5 and Prior 2 in Fig. 6 .

We also evaluated the BOGP performance in a dynamic 
setting where the average cell load varies over time. Using the notation of Section III-C the network state $\theta(t)$ is represented by the cell load at time $t$. The state $\theta$ varies according to a periodic pattern of period 2, namely at each sampling time $t_{n}$ the average cell load alternates between $K=4$ and $K=16$ UEs per TTI. We still used a Matérn kernel for computing the covariance between different RRM configurations, while we employed the Exp-Sine-Squared kernel (11) as time kernel. We let BOGP optimize autonomously and in an online fashion the length scale $\ell_{E S S}$ of the time kernel-regulating the covariance between off-period samples-as described in Section III-D In Figure 7 we illustrate the convergence of the classic static BOGP (non-stationary, time kernel period $D=1$, in the legend) and the dynamic and periodic BOGP $(D=2)$, where each point is averaged over 16 independent runs with random initialization points and a constant prior mean. As a benchmark, we also show the case where two BOGPs are run in parallel (stationary case, in Figure 7), one in each state with $K=4,16$, respectively. Clearly, the latter scenario is implementable only if the state evolution is known in advance. We set the UCB parameter $\beta_{n}=1$ for each iteration $n$ (cfr. Eq. (20)). Classic BO is agnostic to the time evolution and finds a single RRM configuration with good performance in both states, while dynamic BO manages to distinguish between the two scenarios and adapts its RRM configuration accordingly. Letting dynamic BO $(D=2)$ optimize the time length scale $\ell_{E S S}$ produces two effects: i) dynamic $\mathrm{BO}$ suffers from cold start, while it figures out the best value for $\ell_{E S S}$; ii) from iteration 25 onward, the dynamic BO outperforms most of the other approaches since it exploits the correlation between the two scenarios to learn a better configuration for the less loaded scenario $(K=4)$. We think that the gains will be even more accentuated in network deployments with larger load variations (e.g., shopping malls). We remark that in practical RRM settings, network traffic shows seasonal patterns whose periodicity can be inferred with good confidence via historical data and is typically circadian.

\section{CONCLUSIONS AND FUTURE DIRECTIONS}

We provided an accessible introduction to Bayesian optimization with Gaussian Processes (BOGP). Moreover we showed under which conditions BOGP can serve the purpose of tuning RRM parameters in online fashion, by minimizing the risk of incurring performance drops. We instantiated BOGP on a realistic uplink power control setting, where the goal is to optimize two network-wide parameters describing the target received power at the base station and the path loss compensation factor.

We believe that BOGP is a powerful and versatile tool that has not yet received the attention that it deserves from our community. We think that there exist at least three research directions that can pave the way for the adoption of BOGP over a larger set of RRM use cases. The first one is the incorporation of hard constraints-typically, on the user Quality of Service- that are also modeled as black-box functions that can only be learned via noisy sampling. We deem the recent work in [35] as an excellent starting point. Another relevant direction, extending our dynamic BO analysis, consists in directly embedding the "state" of the network in the GP to efficiently transfer the learning across different states, as first studied in [36]. Last but not least, if sufficient historical data is available, a parametric model could be built (in contrast to GPs, which are non-parametric) that is well representative of the objective function. Different Bayesian techniques such as Thompson sampling can be used to find the next point to sample, as reported, e.g., in [8].

\section{ACKNOWLEDGMENTS}

We would like to thank our colleagues Véronique Capdevielle, Richa Gupta, and Suresh Kalyanasundaram for insightful discussions on the topic of OLPC and the pros \& cons of BOGP for RRM.

\section{REFERENCES}

[1] H. Viswanathan and P. E. Mogensen, "Communications in the 6G era," IEEE Access, vol. 8, pp. $57063-57$ 074, 2020.

[2] M. E. Morocho-Cayamcela, H. Lee, and W. Lim, "Machine learning for 5G/B5G mobile and wireless communications: potential, limitations, and future directions," IEEE Access, vol. 7, pp. 137 184-137 206, Sep. 2019.

[3] K. B. Letaief, W. Chen, Y. Shi, J. Zhang, and Y.-J. A. Zhang, "The roadmap to 6G: AI empowered wireless networks," IEEE Communications Magazine, vol. 57, no. 8, pp. 84-90, 2019.

[4] C. Benzaid and T. Taleb, "AI-driven zero touch network and service management in 5G and beyond: challenges and research directions," IEEE Network, vol. 34, no. 2, pp. 186-194, 2020.

[5] N. Kato, B. Mao, F. Tang, Y. Kawamoto, and J. Liu, "Ten challenges in advancing machine learning technologies toward 6G," IEEE Wireless Communications, vol. 27, no. 3, pp. 96-103, 2020.

[6] F. D. Calabrese, L. Wang, E. Ghadimi, G. Peters, L. Hanzo, and P. Soldati, "Learning radio resource management in RANs: Framework, opportunities, and challenges," IEEE Communications Magazine, vol. 56, no. 9, pp. 138-145, 2018.

[7] R. M. Dreifuerst, S. Daulton, Y. Qian, P. Varkey, M. Balandat, S. Kasturia, A. Tomar, A. Yazdan, V. Ponnampalam, and R. W. Heath, "Optimizing coverage and capacity in cellular networks using machine learning," 2020. [Online]. Available: http://arxiv.org/abs/2010.13710

[8] B. Shahriari, K. Swersky, Z. Wang, R. P. Adams, and N. De Freitas, "Taking the human out of the loop: A review of Bayesian optimization," Proceedings of the IEEE, vol. 104, no. 1, pp. 148-175, 2016.

[9] P. I. Frazier, "A tutorial on Bayesian optimization," arXiv preprint arXiv:1807.02811. 2018.

[10] D. Cornford, I. Nabney, and C. Williams, "Adding constrained discontinuities to Gaussian process models of wind fields," Advances in Neural Information Processing Systems, vol. 11, pp. 861-867, 1998.

[11] N. Cesa-Bianchi and G. Lugosi, Prediction, learning, and games. Cambridge University Press, 2006.

[12] R. S. Sutton and A. G. Barto, Reinforcement learning: An introduction. MIT press, 2018.

[13] J. Garcia and F. Fernández, "A comprehensive survey on safe reinforcement learning," Journal of Machine Learning Research, vol. 16, no. 1, pp. 1437-1480, 2015.

[14] A. R. Conn, K. Scheinberg, and L. N. Vicente, Introduction to derivativefree optimization. SIAM, 2009.

[15] C. Audet and W. Hare, Derivative-free and blackbox optimization. Springer, 2017.

[16] C. K. Williams and C. E. Rasmussen, Gaussian processes for machine learning. MIT press Cambridge, MA, 2006, vol. 2, no. 3.

[17] S. Theodoridis, Machine learning: a Bayesian and optimization perspective. Academic press, 2015.

[18] A. Shah, A. Wilson, and Z. Ghahramani, "Student- $t$ processes as alternatives to Gaussian processes," in Artificial intelligence and statistics, 2014, pp. 877-885.

[19] F. M. Nyikosa, M. A. Osborne, and S. J. Roberts, "Bayesian optimization for dynamic problems," arXiv preprint arXiv:1803.03432. 2018.

[20] M. Blum and M. A. Riedmiller, "Optimization of Gaussian process hyperparameters using Rprop," in ESANN. Citeseer, 2013, pp. 339-344. 
[21] R. Pautrat, K. Chatzilygeroudis, and J.-B. Mouret, "Bayesian optimization with automatic prior selection for data-efficient direct policy search," in 2018 IEEE International Conference on Robotics and Automation (ICRA). IEEE, 2018, pp. 7571-7578.

[22] M. Avriel, Nonlinear programming: Analysis and methods. Courier Corporation, 2003.

[23] M. L. Puterman, Markov decision processes: Discrete stochastic dynamic programming. John Wiley \& Sons, 2014.

[24] W. B. Powell, Approximate Dynamic Programming: Solving the curses of dimensionality. John Wiley \& Sons, 2007, vol. 703.

[25] J. Močkus, "On Bayesian methods for seeking the extremum," in Optimization techniques IFIP technical conference. Berlin, Heidelberg: Springer Berlin Heidelberg, 1975, pp. 400-404.

[26] D. R. Jones, M. Schonlau, and W. J. Welch, "Efficient global optimization of expensive black-box functions," Journal of Global optimization, vol. 13, no. 4, pp. 455-492, 1998.

[27] D. Huang, T. T. Allen, W. I. Notz, and R. A. Miller, "Sequential kriging optimization using multiple-fidelity evaluations," Structural and Multidisciplinary Optimization, vol. 32, no. 5, pp. 369-382, 2006.

[28] D. C. Liu and J. Nocedal, "On the limited memory BFGS method for large scale optimization," Mathematical programming, vol. 45, no. 1-3, pp. 503-528, 1989.

[29] P. Frazier, W. Powell, and S. Dayanik, "The knowledge-gradient policy for correlated normal beliefs," INFORMS journal on Computing, vol. 21, no. 4, pp. 599-613, 2009 .

[30] J. Wu and P. Frazier, "The parallel knowledge gradient method for batch Bayesian optimization," Advances in Neural Information Processing Systems, vol. 29, pp. 3126-3134, 2016.

[31] N. Srinivas, A. Krause, S. M. Kakade, and M. W. Seeger, "Gaussian process optimization in the bandit setting: No regret and experimental design," in Proceedings of the 27th International Conference on Machine Learning (ICML-10), June 21-24, 2010, Haifa, Israel, J. Fürnkranz and T. Joachims, Eds. Omnipress, 2010, pp. 1015-1022. [Online]. Available: https://icml.cc/Conferences/2010/papers/422.pdf

[32] R. Müllner, C. F. Ball, K. Ivanov, J. Lienhart, and P. Hric, "Uplink power control performance in UTRAN LTE networks," Lecture Notes in Electrical Engineering, vol. 41 LNEE, pp. 175-184, 2009.

[33] A. Haider, R. S. Sinha, and S. H. Hwang, "Investigation of open-loop transmit power control parameters for homogeneous and heterogeneous small-cell uplinks," ETRI Journal, vol. 40, no. 1, pp. 51-60, feb 2018.

[34] J. Mo and J. Walrand, "Fair end-to-end window-based congestion control," IEEE/ACM Transactions on Networking, vol. 8, no. 5, pp. 556567 , oct 2000.

[35] B. Letham, B. Karrer, G. Ottoni, and E. Bakshy, "Constrained Bayesian Optimization with Noisy Experiments," Bayesian Analysis, vol. 14, no. 2, pp. 495 - 519, 2019. [Online]. Available: https://doi.org/10.1214/18-BA1110

[36] A. Krause and C. Ong, "Contextual Gaussian Process Bandit Optimization," in Advances in Neural Information Processing Systems, J. Shawe-Taylor, R. Zemel, P. Bartlett, F. Pereira, and K. Q. Weinberger, Eds., vol. 24. Curran Associates, Inc. 2011. [Online]. Available: https://proceedings.neurips.cc/paper/2011/ file/f3f1b7fc5a8779a9e618e1f23a7b7860-Paper.pdf 